\title{
ZERO-CYCLES ON GARBAGNATI SURFACES
}

\author{
ROBERT LATERVEER
}

\begin{abstract}
Garbagnati has constructed certain surfaces of general type that are bidouble planes as well as double covers of K3 surfaces. In this note, we study the Chow groups (and Chow motive) of these surfaces.
\end{abstract}

\section{INTRODUCTION}

In [8], Garbagnati studies K3 surfaces which admit a double cover. In the course of this work, she constructs certain surfaces of general type that are birational to bidouble covers of $\mathbb{P}^{2}$ and that we propose to call Garbagnati surfaces. These surfaces have the remarkable property of being "K3 burgers", i.e. they are of geometric genus $m:=p_{g}(S) \in\{1,2,3\}$ and their transcendental cohomology splits

$$
H_{t r}^{2}(S, \mathbb{Q})=\bigoplus_{j=1}^{m} H_{t r}^{2}\left(T_{j}, \mathbb{Q}\right),
$$

where the $T_{j}$ are K3 surfaces. (For other examples of K3 burgers, cf. [6], [18].)

The main result of the present note is that this "K3 burger" relation is also valid on the level of Chow groups (and Chow motives):

Theorem (=Theorem 3.1). Let $S$ be a Garbagnati surface. Let $T_{1}, \ldots, T_{m}$ be the associated $K 3$ surfaces where $m=p_{g}(S)$. There is an isomorphism of Chow groups

$$
A_{\text {hom }}^{2}(S) \stackrel{\cong}{\rightarrow} \bigoplus_{j=1}^{m} A_{\text {hom }}^{2}\left(T_{j}\right) .
$$

Moreover, there is an isomorphism of Chow motives

$$
h_{t r}^{2}(S) \cong \bigoplus_{j=1}^{m} h_{t r}^{2}\left(T_{j}\right) \text { in } \mathcal{M}_{\mathrm{rat}}
$$

(Here, $h_{t r}^{2}()$ denotes the transcendental part of the motive, $c f .92 .3$ )

This is in agreement with the Bloch-Beilinson conjectures [11]. As a consequence of the main result, one can verify an old conjecture of Voisin [28] for certain Garbagnati surfaces:

2010 Mathematics Subject Classification. Primary 14C15, 14C25, 14C30, 14J28, 14J29.

Key words and phrases. Algebraic cycles, Chow group, motive, Bloch-Beilinson filtration, surface of general type, K3 surface. 
Corollary (=Corollary 4.2). Let $S$ be a Garbagnati surface of type G1, G2b or G3 (cf. \$2.1). Let $n$ be an integer strictly larger than the geometric genus $p_{g}(S)$. Then for any degree 0 0-cycles $a_{1}, \ldots, a_{n} \in A^{2}(S)_{\mathbb{Z}}$, one has

$$
\sum_{\sigma \in \mathfrak{S}_{n}} \operatorname{sgn}(\sigma) a_{\sigma(1)} \times \cdots \times a_{\sigma(n)}=0 \text { in } A^{2 n}\left(S^{n}\right)_{\mathbb{Z}} .
$$

(Here $\mathfrak{S}_{n}$ is the symmetric group on $n$ elements, and $\operatorname{sgn}(\sigma)$ is the sign of the permutation $\sigma$. The notation $a_{1} \times \cdots \times a_{n}$ is shorthand for the 0 -cycle $\left(p_{1}\right)^{*}\left(a_{1}\right) \cdot\left(p_{2}\right)^{*}\left(a_{2}\right) \cdots\left(p_{n}\right)^{*}\left(a_{n}\right)$ on $S^{n}$, where the $p_{j}: S^{n} \rightarrow S$ are the various projections.)

For surfaces of type G1, this was already proven in [15, Proposition 29], but the present argument seems easier and more conceptual.

Another consequence is a "motivic Torelli theorem" for Garbagnati surfaces:

Corollary (=Corollary 4.4). Let $S$ and $S^{\prime}$ be two Garbagnati surfaces, and assume $S$ and $S^{\prime}$ are isometric (i.e., there is an isomorphism of $\mathbb{Q}$-vector spaces $H_{t r}^{2}(S, \mathbb{Q}) \cong H_{t r}^{2}\left(S^{\prime}, \mathbb{Q}\right)$ compatible with Hodge structures and cup product). Then there is an isomorphism of Chow motives

$$
h_{t r}^{2}(S) \cong h_{t r}^{2}\left(S^{\prime}\right) \text { in } \mathcal{M}_{\text {rat }} .
$$

Other consequences are Kimura finite-dimensionality for certain Garbagnati surfaces (Corollary 4.5), and a "relative Bloch conjecture" type of result concerning the action of certain automorphisms (Corollary 4.6).

Conventions. In this article, the word variety will refer to a reduced irreducible scheme of finite type over $\mathbb{C}$. A subvariety is a (possibly reducible) reduced subscheme which is equidimensional.

All Chow groups will be with rational coefficients: we will denote by $A_{j}(X)$ the Chow group of $j$-dimensional cycles on $X$ with $\mathbb{Q}$-coefficients; for $X$ smooth of dimension $n$ the notations $A_{j}(X)$ and $A^{n-j}(X)$ are used interchangeably.

The notations $A_{\text {hom }}^{j}(X), A_{A J}^{j}(X)$ will be used to indicate the subgroups of homologically trivial, resp. Abel-Jacobi trivial cycles. For a morphism $f: X \rightarrow Y$, we will write $\Gamma_{f} \in$ $A_{*}(X \times Y)$ for the graph of $f$. The contravariant category of Chow motives (i.e., pure motives with respect to rational equivalence as in [26], [23]) will be denoted $\mathcal{M}_{\text {rat }}$.

\section{PRELiminaries}

\subsection{Garbagnati surfaces.}

Definition 2.1 ([8]). A surface of type G1 is a surface $X$ as in [8, Proposition 6.1]. A surface if type $G 1$ has $p_{g}=1$, and is birational to a bidouble cover of $\mathbb{P}^{2}$ branched along two cubics and a line.

A surface of type G2a is a surface $X_{6}^{(1)}$ as in [8, Section 5.4]. A surface of type G2a has $p_{g}=2$, and is birational to a bidouble cover of $\mathbb{P}^{2}$ branched along a quintic and two lines.

A surface of type $G 2 b$ is a surface $X_{9}^{(2)}$ as in [8, Section 5.4]. A surface of type G2b has $p_{g}=2$, and is birational to a bidouble cover of $\mathbb{P}^{2}$ branched along a quartic and two quadrics. 
A surface of type G3 is a surface $Z$ as in [8, Proposition 6.1]. A surface of type G3 has $p_{g}=3$, and is birational to a bidouble cover of $\mathbb{P}^{2}$ branched along three cubics.

Remark 2.2. For details on the construction of bidouble covers, cf. [5].

The bidouble covers birational to surfaces of type G1 are also known as special Kunev surfaces, and are studied (particularly in relation to Torelli problems, where they provide counterexamples) in [4], [14], [27].

The bidouble covers birational to surfaces of type G2a are also called special Horikawa surfaces. These surfaces are studied in [24], where it is shown they satisfy generic global Torelli.

\subsection{Quotient varieties.}

Definition 2.3. A projective quotient variety is a variety

$$
X=Y / G \text {, }
$$

where $Y$ is a smooth projective variety and $G \subset A u t(Y)$ is a finite group.

Proposition 2.4 (Fulton [7]). Let $X$ be a projective quotient variety of dimension $n$. Let $A^{*}(X)$ denote the operational Chow cohomology ring with $\mathbb{Q}$-coefficients. The natural map

$$
A^{i}(X) \rightarrow A_{n-i}(X)
$$

is an isomorphism for all $i$.

Proof. This is [7, Example 17.4.10].

Remark 2.5. It follows from Proposition 2.4 that the formalism of correspondences goes through unchanged for projective quotient varieties (this is also noted in [7, Example 16.1.13]). We can thus consider motives $(X, p, 0) \in \mathcal{M}_{\text {rat }}$, where $X$ is a projective quotient variety and $p \in$ $A^{n}(X \times X)$ is a projector. For a projective quotient variety $X=Y / G$, one readily proves (using Manin's identity principle) that there is an isomorphism

$$
h(X) \cong h(Y)^{G}:=\left(Y, \Delta_{Y}^{G}, 0\right) \text { in } \mathcal{M}_{\text {rat }},
$$

where $\Delta_{Y}^{G}$ denotes the idempotent $\frac{1}{|G|} \sum_{g \in G} \Gamma_{g}$.

\subsection{Transcendental part of the motive.}

Theorem 2.6 (Kahn-Murre-Pedrini [12]). Let $S$ be any smooth projective surface, and let $h(X) \in \mathcal{M}_{\text {rat }}$ denote the Chow motive of $S$. There exists a self-dual Chow-Künneth decomposition $\left\{\pi_{S}^{i}\right\}$ of $S$, with the property that there is a further splitting in orthogonal idempotents

$$
\pi_{S}^{2}=\pi_{S}^{2, a l g}+\pi_{S}^{2, t r} \text { in } A^{2}(S \times S) .
$$

The action on cohomology is

$$
\left(\pi_{S}^{2, a l g}\right)_{*} H^{*}(S, \mathbb{Q})=N^{1} H^{2}(S, \mathbb{Q}), \quad\left(\pi_{S}^{2, t r}\right)_{*} H^{*}(S, \mathbb{Q})=H_{t r}^{2}(S, \mathbb{Q}),
$$

where the transcendental cohomology $H_{t r}^{2}(S, \mathbb{Q}) \subset H^{2}(S, \mathbb{Q})$ is defined as the orthogonal complement of $N^{1} H^{2}(S, \mathbb{Q})$ with respect to the intersection pairing. The action on Chow groups is

$$
\left(\pi_{S}^{2, a l g}\right)_{*} A^{*}(S)=N^{1} H^{2}(S, \mathbb{Q}), \quad\left(\pi_{S}^{2, t r}\right)_{*} A^{*}(S)=A_{A J}^{2}(S) .
$$


This gives rise to a well-defined Chow motive

$$
h_{t r}^{2}(S):=\left(S, \pi_{S}^{2, t r}, 0\right) \subset h(X) \in \mathcal{M}_{\text {rat }},
$$

the so-called transcendental part of the motive of $S$.

Proof. Let $\left\{\pi_{S}^{i}\right\}$ be a Chow-Künneth decomposition as in [12, Proposition 7.2.1]. The assertion then follows from [12, Proposition 7.2.3]. The projector $\pi_{S}^{2, a l g}$ is of the form

$$
\pi_{S}^{2, a l g}=\sum_{j=1}^{\rho} D_{j} \times D_{j}^{\prime},
$$

where $D_{1}, \ldots, D_{\rho}$ is a basis for the Néron-Severi group of $S$, and $D_{1}^{\prime}, \ldots, D_{\rho}^{\prime}$ is a dual basis.

\section{MAIN RESULT}

Theorem 3.1. Let $S$ be a Garbagnati surface. Let $T_{1}, \ldots, T_{m}$ be the associated $K 3$ surfaces where $m=p_{g}(S)$. There is an isomorphism

$$
A_{\text {hom }}^{2}(S) \stackrel{\cong}{\rightarrow} \bigoplus_{j=1}^{m} A_{\text {hom }}^{2}\left(T_{j}\right) .
$$

Moreover, one can choose Chow-Künneth decompositions for $S$ and $T_{j}$ such that there is an isomorphism of Chow motives

$$
h_{t r}^{2}(S) \cong \bigoplus_{j=1}^{m} h_{t r}^{2}\left(T_{j}\right) \text { in } \mathcal{M}_{\text {rat }} .
$$

Proof. The statement being birationally invariant, we may assume $S$ is a bidouble plane as described in Definition 2.1. From the construction of $S$ as a bidouble plane, it follows that there are three commuting involutions $\sigma_{i} \in \operatorname{Aut}(S), i=1,2,3$, such that

$$
\sigma_{3}=\sigma_{1} \circ \sigma_{2}=\sigma_{2} \circ \sigma_{1} \text {. }
$$

Let $Q_{i}:=S /\left\langle\sigma_{i}\right\rangle$ denote the quotients, so there is a commuting diagram (where the arrows are the various quotient maps)

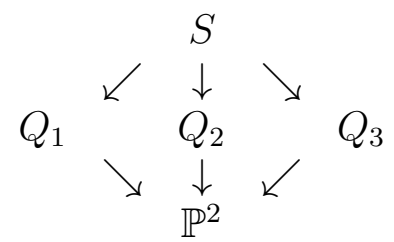

Define $A^{2}(S)^{ \pm \mp}$ as the subgroup of $A^{2}(S)$ where $\left(\sigma_{1}, \sigma_{2}\right)$ acts as $( \pm 1, \mp 1)$. There is a decomposition

$$
A_{\text {hom }}^{2}(S)=A_{\text {hom }}^{2}(S)^{++} \oplus A_{\text {hom }}^{2}(S)^{+-} \oplus A_{\text {hom }}^{2}(S)^{-+} \oplus A_{\text {hom }}^{2}(S)^{--} .
$$

The summand $A_{\text {hom }}^{2}(S)^{++}$, being isomorphic to $A_{h o m}^{2}\left(\mathbb{P}^{2}\right)$, is zero, while the three other summands are isomorphic to $A_{h o m}^{2}\left(Q_{i}\right), i=1,2,3$. Thus we get a natural isomorphism

$$
A_{\text {hom }}^{2}(S) \cong A_{\text {hom }}^{2}\left(Q_{1}\right) \oplus A_{\text {hom }}^{2}\left(Q_{2}\right) \oplus A_{\text {hom }}^{2}\left(Q_{3}\right) \text {. }
$$


In case the surface $S$ is of type G3, there are 3 associated $\mathrm{K} 3$ surfaces $T_{j}$ and one has natural isomorphisms

$$
A_{\text {hom }}^{2}\left(Q_{j}\right) \cong A_{\text {hom }}^{2}\left(T_{j}\right)
$$

(indeed, the $T_{j}$ are resolutions of singularities of the $Q_{j}$, and cyclic quotient singularities can be resolved by strings of rational curves, cf. [1]; the isomorphism then follows from [22, Proposition 1.7]). This proves the statement for Chow groups.

In case $S$ is of type $\mathrm{G} 2 \mathrm{a}$ or $\mathrm{G} 2 \mathrm{~b}$, two of the $Q_{j}$ (say $Q_{1}$ and $Q_{2}$ ) are birational to a $\mathrm{K} 3$ surface, and the third surface $Q_{3}$ is rational, hence $A_{h o m}^{2}\left(Q_{3}\right)=0$. This proves the statement for Chow groups for cases $\mathrm{G} 2 \mathrm{a}, \mathrm{G} 2 \mathrm{~b}$.

Finally, for the case $\mathrm{G} 1$, one of the $Q_{j}$ (say $Q_{1}$ ) is birational to a $\mathrm{K} 3$ surface, and the other two are rational; the statement for Chow groups follows similarly.

The statement for motives is proven along the same lines, by exploiting the bidouble cover structure: we can define motives $h(S)^{ \pm \mp} \in \mathcal{M}_{\text {rat }}$ by setting

$$
\begin{aligned}
h(S)^{++}:=\left(S, \frac{1}{4}\left(\Delta_{S}+\Gamma_{\sigma_{1}}\right) \circ\left(\Delta_{S}+\Gamma_{\sigma_{2}}\right), 0\right), \\
h(S)^{+-}:=\left(S, \frac{1}{4}\left(\Delta_{S}+\Gamma_{\sigma_{1}}\right) \circ\left(\Delta_{S}-\Gamma_{\sigma_{2}}\right), 0\right), \\
h(S)^{-+}:=\left(S, \frac{1}{4}\left(\Delta_{S}-\Gamma_{\sigma_{1}}\right) \circ\left(\Delta_{S}+\Gamma_{\sigma_{2}}\right), 0\right), \\
h(S)^{--}:=\left(S, \frac{1}{4}\left(\Delta_{S}-\Gamma_{\sigma_{1}}\right) \circ\left(\Delta_{S}-\Gamma_{\sigma_{2}}\right), 0\right) .
\end{aligned}
$$

(It is readily checked the given cycles are idempotents and so define motives.)

This gives a decomposition

$$
h(S)=h(S)^{++} \oplus h(S)^{+-} \oplus h(S)^{-+} \oplus h(S)^{--} \text {in } \mathcal{M}_{\text {rat }} .
$$

Defining $h^{0}(S)$ and $h^{4}(S)$ by the choice of a zero-cycle invariant under $\sigma_{1}$ and $\sigma_{2}$, we get a similar decomposition for $h^{2}(S)=h(S)-h^{0}(S)-h^{4}(S)$. Next, we can choose a basis $D_{1}, \ldots, D_{\rho}$ for the Néron-Severi group of $S$ such that $D_{1}, \ldots, D_{\rho_{1}}$ are of type ++ (i.e. they are invariant under $\sigma_{1}$ and $\left.\sigma_{2}\right), D_{\rho_{1}+1}, \ldots, D_{\rho_{2}}$ are of type $+-, D_{\rho_{2}+1}, \ldots, D_{\rho_{3}}$ are of type -+ , and the remaining divisors are of type - - . The dual basis decomposes similarly (a divisor $D_{j}$ and its dual $D_{j}^{\prime}$ are of the same type), and so we get a decomposition

$$
h_{\text {alg }}^{2}(S)=h_{\text {alg }}^{2}(S)^{++} \oplus h_{\text {alg }}^{2}(S)^{+-} \oplus h_{\text {alg }}^{2}(S)^{-+} \oplus h_{\text {alg }}^{2}(S)^{--} \text {in } \mathcal{M}_{\text {rat }} .
$$

It follows that there is an induced decomposition

$$
h_{t r}^{2}(S)=h_{t r}^{2}(S)^{++} \oplus h_{t r}^{2}(S)^{+-} \oplus h_{t r}^{2}(S)^{-+} \oplus h_{t r}^{2}(S)^{--} \text {in } \mathcal{M}_{\text {rat }} .
$$

The first summand $h_{t r}^{2}(S)^{++}$is the transcendental part of the motive of $\mathbb{P}^{2}$, which is zero. For surfaces $S$ of type G3, the remaining three summands are isomorphic to $h_{t r}^{2}\left(S /\left\langle\sigma_{j}\right\rangle\right)=h_{t r}^{2}\left(T_{j}\right)$ where $T_{j}$ is an associated $\mathrm{K} 3$ surface. In cases G2a and G2b, the summand $h_{t r}^{2}(S)^{--}$corresponds to $h_{t r}^{2}\left(Q_{3}\right)$, which is zero as $Q_{3}$ is a rational surface. Finally, in case $\mathrm{G} 1$ there are two rational surfaces $Q_{2}$ and $Q_{3}$ and thus

$$
h_{t r}^{2}(S)=h_{t r}^{2}(S)^{+-}=h_{t r}^{2}\left(Q_{1}\right)=h_{t r}^{2}\left(T_{1}\right) \text { in } \mathcal{M}_{\text {rat }} .
$$


This proves the motivic statement.

\section{SOME CONSEQUENCES}

\subsection{Voisin conjecture.}

Conjecture 4.1 (Voisin [28]). Let $S$ be a smooth projective surface. Let $n$ be an integer strictly larger than the geometric genus $p_{g}(S)$. Then for any 0 -cycles $a_{1}, \ldots, a_{n} \in A_{A J}^{2}(S)_{\mathbb{Z}}$, one has

$$
\sum_{\sigma \in \mathfrak{S}_{n}} \operatorname{sgn}(\sigma) a_{\sigma(1)} \times \cdots \times a_{\sigma(n)}=0 \text { in } A^{2 n}\left(S^{n}\right)_{\mathbb{Z}} .
$$

(Here $\mathfrak{S}_{n}$ is the symmetric group on $n$ elements, and $\operatorname{sgn}(\sigma)$ is the sign of the permutation $\sigma$. The notation $a_{1} \times \cdots \times a_{n}$ is shorthand for the 0 -cycle $\left(p_{1}\right)^{*}\left(a_{1}\right) \cdot\left(p_{2}\right)^{*}\left(a_{2}\right) \cdots\left(p_{n}\right)^{*}\left(a_{n}\right)$ on $S^{n}$, where the $p_{j}: S^{n} \rightarrow S$ are the various projections.)

This conjecture is a particular instance of the Bloch-Beilinson conjectures. For surfaces of geometric genus 0, conjecture 4.1 reduces to Bloch's conjecture [3]. As for geometric genus 1 , Voisin's conjecture is still open for a general K3 surface; examples of surfaces of geometric genus 1 verifying the conjecture are given in [28], [15], [16], [17]. Examples of surfaces with geometric genus strictly larger than 1 verifying the conjecture are given in [19], [20], [21].

Corollary 4.2. Let $S$ be a surface of type G1, G2b or G3. Then Voisin's conjecture is true for $S$.

Proof. Thanks to Roitman's theorem, Voisin's conjecture is equivalent to the version with $\mathbb{Q}$ coefficients. Voisin's conjecture (for an arbitrary surface $S$ ) can then be succinctly restated as

$$
A_{0}\left(\wedge^{n} h_{t r}^{2}(S)\right)=0 \text { for all } n>p_{g}(S),
$$

where the wedge product of a motive is as defined in [13, Definition 3.5].

Let $S$ now be a Garbagnati surface, and let $T_{1}, \ldots, T_{m}$ denote the K3 surfaces associated to $S$. Invoking Theorem 3.1, we find that Voisin's conjecture for $S$ is implied by Voisin's conjecture for $T_{1}, \ldots, T_{m}$. Indeed, we have

$$
\begin{aligned}
A_{0}\left(\wedge^{n} h_{t r}^{2}(S)\right) & =A_{0}\left(\wedge^{n}\left(\bigoplus_{j=1}^{m} h_{t r}^{2}\left(T_{j}\right)\right)\right) \\
& =A_{0}\left(\bigoplus_{n_{1}+\cdots+n_{m}=n} \wedge^{n_{1}} h_{t r}^{2}\left(T_{1}\right) \otimes \cdots \otimes \wedge^{n_{m}} h_{t r}^{2}\left(T_{m}\right)\right) \\
& =\bigoplus_{n_{1}+\cdots+n_{m}=n} A_{0}\left(\wedge^{n_{1}} h_{t r}^{2}\left(T_{1}\right)\right) \otimes \cdots \otimes A_{0}\left(\wedge^{n_{m}} h_{t r}^{2}\left(T_{m}\right)\right) .
\end{aligned}
$$

Assume now that $n>m:=p_{g}(S)$. Then in each summand there is an $n_{j}>1$. Hence assuming Voisin's conjecture for all the $T_{j}$, each summand vanishes, and so Voisin's conjecture holds for $S$ as claimed.

To finish the proof, it remains to observe that Voisin's conjecture is known for K3 surfaces obtained by desingularizing double planes branched along the union of two cubics [28, Theorem 3.4], and also for K3 surfaces obtained from double planes branched along a quartic and a quadric [15, Proposition 14]. 
Remark 4.3. We do not know whether Voisin's conjecture holds for surfaces of type G2a. The reason is that Voisin's conjecture is not yet known for $K 3$ double planes branched along a quintic and a line (and the method of [28, Theorem 3.4] and [15, Proposition 14] seems ill-suited for this case).

\subsection{Motivic Torelli.}

Corollary 4.4. Let $S$ and $S^{\prime}$ be two Garbagnati surfaces, and assume $S$ and $S^{\prime}$ are isometric (i.e., there is an isomorphism of $\mathbb{Q}$-vector spaces $H_{t r}^{2}(S, \mathbb{Q}) \cong H_{t r}^{2}\left(S^{\prime}, \mathbb{Q}\right)$ compatible with Hodge structures and cup product). Then there is an isomorphism of Chow motives

$$
h_{t r}^{2}(S) \cong h_{t r}^{2}\left(S^{\prime}\right) \text { in } \mathcal{M}_{\text {rat }} .
$$

Proof. This is a direct transplantation of the analogous result for K3 surfaces.

Let $T_{j}$ and $T_{j}^{\prime}$ denote the $\mathrm{K} 3$ surfaces associated to $S$ resp. to $S^{\prime}$, for $j=1, \ldots, m:=$ $p_{g}(S)=p_{g}\left(S^{\prime}\right)$. The image of $H_{t r}^{2}\left(T_{j}, \mathbb{Q}\right) \subset H_{t r}^{2}(S, \mathbb{Q})$ under the isometry $\phi$ is a sub-Hodge structure of $H_{t r}^{2}\left(S^{\prime}, \mathbb{Q}\right)$. Since the transcendental cohomology of K3 surfaces are indecomposable Hodge structures, the image must be equal to one of the $H_{t r}^{2}\left(T_{j}^{\prime}, \mathbb{Q}\right)$, and so we may assume that $\phi\left(H_{t r}^{2}\left(T_{j}, \mathbb{Q}\right)\right)=H_{t r}^{2}\left(T_{j}^{\prime}, \mathbb{Q}\right)$. The inclusion $H_{t r}^{2}\left(T_{j}, \mathbb{Q}\right) \subset H_{t r}^{2}(S, \mathbb{Q})$ is given by the composition

$$
H_{t r}^{2}\left(T_{j}, \mathbb{Q}\right) \stackrel{\cong}{\longleftarrow} H_{t r}^{2}\left(S /\left\langle\sigma_{j}\right\rangle, \mathbb{Q}\right) \stackrel{\left(p_{j}\right)^{*}}{\longrightarrow} H_{t r}^{2}(S, \mathbb{Q}),
$$

where the left arrow is induced by a resolution of singularities. This shows that the inclusion $H_{t r}^{2}\left(T_{j}, \mathbb{Q}\right) \subset H_{t r}^{2}(S, \mathbb{Q})$ is compatible with cup product. Hence, the isometry $\phi$ decomposes as a sum

$$
\phi=\sum_{j=1}^{m} \phi_{j}: \bigoplus_{j=1}^{m} H_{t r}^{2}\left(T_{j}, \mathbb{Q}\right) \rightarrow \bigoplus_{j=1}^{m} H_{t r}^{2}\left(T_{j}^{\prime}, \mathbb{Q}\right),
$$

where each $\phi_{j}$ is an isometry. Huybrechts' result [10] then guarantees that there are isomorphisms of Chow motives

$$
h_{t r}^{2}\left(T_{j}\right) \cong h_{t r}^{2}\left(T_{j}^{\prime}\right) \text { in } \mathcal{M}_{\text {rat }}(j=1, \ldots, m) .
$$

Applying Theorem 3.1, it follows that there is an isomorphism

$$
h_{t r}^{2}(S) \cong h_{t r}^{2}\left(S^{\prime}\right) \text { in } \mathcal{M}_{\text {rat }} \text {. }
$$

\subsection{Finite-dimensionality.}

Corollary 4.5. The following surfaces have finite-dimensional motive (in the sense of Kimura [13]):

(1) Surfaces of type $G 1$ with $\operatorname{dim} H_{t r}^{2}(S, \mathbb{Q}) \leq 3$;

(2) Surfaces of type $G 2$ with $\operatorname{dim} H_{t r}^{2}(S, \mathbb{Q}) \leq 5$;

(3) Surfaces of type $G 3$ with $\operatorname{dim} H_{t r}^{2}(S, \mathbb{Q}) \leq 7$. 
Proof. Let $m \in\{1,2,3\}$ denote the geometric genus $m:=p_{g}(S)$, and let $T_{1}, \ldots, T_{m}$ be the associated K3 surfaces. Recall that there is an isomorphism

$$
H_{t r}^{2}(S, \mathbb{Q}) \cong \oplus_{j=1}^{m} H_{t r}^{2}\left(T_{j}, \mathbb{Q}\right)
$$

The $T_{j}$ being $\mathrm{K} 3$ surfaces, the dimension of $H_{t r}^{2}\left(T_{j}, \mathbb{Q}\right)$ is at least 2 , and so the assumption on $H_{t r}^{2}(S, \mathbb{Q})$ implies that

$$
\operatorname{dim} H_{t r}^{2}\left(T_{j}, \mathbb{Q}\right) \leq 3 \quad(j=1 \ldots, m) .
$$

It follows from [25] that the $T_{j}$ have finite-dimensional motive. In view of the isomorphism of Theorem 3.1, this implies the corollary.

\subsection{Bloch conjecture for automorphisms.}

Corollary 4.6. Let $S$ be a Garbagnati surface with $p_{g}(S)=: m$, and let $\sigma_{1}, \ldots, \sigma_{m}$ be the involutions for which the quotient is birational to a K3 surface. Let $f \in \operatorname{Aut}(S)$ be a finite order automorphism that commutes with $\sigma_{1}, \ldots, \sigma_{m}$, and such that

$$
f^{*}=\mathrm{id}: \quad H^{2,0}(S) \rightarrow H^{2,0}(S) .
$$

Then also

$$
f^{*}=\mathrm{id}: \quad A^{2}(S) \rightarrow A^{2}(S) .
$$

Proof. Since $f$ commutes with the $\sigma_{j}, f$ induces automorphisms $f_{j} \in \operatorname{Aut}\left(T_{j}\right), j=1, \ldots, m$ that are symplectic of finite order. Huybrechts has proven [9] that such automorphisms act as the identity on zero-cycles, i.e. one has

$$
\left(f_{j}\right)^{*}=\mathrm{id}: \quad A^{2}\left(T_{j}\right) \rightarrow A^{2}\left(T_{j}\right) \quad(j=1, \ldots, m) .
$$

Theorem 3.1, combined with the commutative diagram

$$
\begin{array}{ccc}
A_{\text {hom }}^{2}(S) & \stackrel{f^{*}}{\longrightarrow} & A_{\text {hom }}^{2}(S) \\
\uparrow\left(p_{j}\right)^{*} & & \uparrow\left(p_{j}\right)^{*}
\end{array} \quad(j=1, \ldots, m)
$$

implies that

$$
f^{*}=\mathrm{id}: \quad A_{\text {hom }}^{2}(S) \rightarrow A_{\text {hom }}^{2}(S) .
$$

Since the subspace $A^{2}(S)^{++} \cong \mathbb{Q}$ is also fixed by $f$, this proves the corollary.

Acknowledgements. Thanks to Len and Kai and Yoyo for being wonderful collaborators in the 2019 Special Program "Papa Werkt Thuis". 


\section{REFERENCES}

[1] W. Barth, K. Hulek, C. Peters and A. van de Ven, Compact complex surfaces, 2nd edition, Springer-Verlag Berlin 2004,

[2] A. Beauville and C. Voisin, On the Chow ring of a K3 surface, J. Alg. Geom. 13 (2004), 417—426,

[3] S. Bloch, Lectures on algebraic cycles, Duke Univ. Press Durham 1980,

[4] F. Catanese, Surfaces with $K^{2}=p_{g}=1$ and their period mapping, in: Algebraic geometry (Copenhagen, 1978), Springer Lecture Notes in Mathematics, Springer 1979,

[5] F. Catanese, Singular bidouble covers and the construction of interesting algebraic surfaces, Contemporary Mathematics 241 (1999), 97-119,

[6] J. Commelin and M. Penegini, On the cohomology of surfaces with $p_{g}=q=2$ and maximal Albanese dimension, Trans. Amer. Math. Soc. 373 (2020), 1749-1773,

[7] W. Fulton, Intersection theory, Springer-Verlag Ergebnisse der Mathematik, Berlin Heidelberg New York Tokyo 1984,

[8] A. Garbagnati, Smooth double covers of $K 3$ surfaces, Annali della Scu. Norm. di Pisa 19 no. 1 (2019), $345-386$,

[9] D. Huybrechts, Symplectic automorphisms of K3 surfaces of arbitrary order, Math. Res. Letters 19 (4) (2012), 947-951,

[10] D. Huybrechts, Motives of isogenous $K 3$ surfaces, to appear in Comment. Math. Helvetici,

[11] U. Jannsen, Motivic sheaves and filtrations on Chow groups, in: Motives (U. Jannsen et alii, eds.), Proceedings of Symposia in Pure Mathematics Vol. 55 (1994), Part 1,

[12] B. Kahn, J. Murre and C. Pedrini, On the transcendental part of the motive of a surface, in: Algebraic cycles and motives (J. Nagel and C. Peters, editors), Cambridge University Press, Cambridge 2007,

[13] S. Kimura, Chow groups are finite dimensional, in some sense, Math. Ann. 331 (2005), 173-201,

[14] V. Kynev, An example of a simply-connected surface of general type for which the local Torelli theorem does not hold, C. R. Acad. Bulgare Sci. 30 no. 3 (1977), 323-325,

[15] R. Laterveer, Some results on a conjecture of Voisin for surfaces of geometric genus one, Boll. Unione Mat. Italiano 9 no. 4 (2016), 435-452,

[16] R. Laterveer, Algebraic cycles on surfaces with $p_{g}=1$ and $q=2$, Comment. Math. Univ. St. Pauli 65 no. 2 (2016), 121-130,

[17] R. Laterveer, Algebraic cycles and Todorov surfaces, Kyoto Journal of Mathematics 58 no. 3 (2018), $493-527$,

[18] R. Laterveer, Algebraic cycles and triple K3 burgers, Arkiv för Matematik 57 (2019), 157—189,

[19] R. Laterveer, Zero-cycles on self-products of surfaces: some new examples verifying Voisin's conjecture, Rend. Circ. Mat. Palermo, Series 2, 68(2) (2019), 419-431,

[20] R. Laterveer, Zero-cycles on Cancian-Frapporti surfaces, Annali dell'Univ. di Ferrara 65 no. 1 (2019), $127-137$,

[21] R. Laterveer and Ch. Vial, On the Chow ring of Cynk-Hulek Calabi-Yau varieties and Schreieder varieties, Canadian Journal of Math. 72 no. 2 (2020), 505-536,

[22] R. Laterveer, Bloch's conjecture for some numerical Campedelli surfaces, to appear in Asian Journal of Math.,

[23] J. Murre, J. Nagel and C. Peters, Lectures on the theory of pure motives, Amer. Math. Soc. University Lecture Series 61, Providence 2013,

[24] G. Pearlstein and Z. Zhang, A generic global Torelli theorem for certain Horikawa surfaces, Algebraic Geometry 6 (2) (2019), 132-147,

[25] C. Pedrini, On the finite dimensionality of a K3 surface, Manuscripta Mathematica 138 (2012), 59-72,

[26] T. Scholl, Classical motives, in: Motives (U. Jannsen et alii, eds.), Proceedings of Symposia in Pure Mathematics Vol. 55 (1994), Part 1,

[27] A. Todorov, Surfaces of general type with $p_{g}=1$ and $(K, K)=1$, Ann. Sci. de l'Ecole Normale Sup. 13 (1980), 1-21, 
[28] C. Voisin, Remarks on zero-cycles of self-products of varieties, in: Moduli of vector bundles, Proceedings of the Taniguchi Congress (M. Maruyama, ed.), Marcel Dekker New York Basel Hong Kong 1994,

Institut de Recherche Mathématique Avancée, CNRS - Université de Strasbourg, 7 Rue RENÉ Descartes, 67084 STRASBOURG CEDEX, FRANCE.

E-mail address: robert. laterveer@math.unistra.fr 